\title{
Recent Results from H1 Experiment at HERA
}

\author{
Cristinel Diaconu * \\ Centre de Physique des Particules de Marseille \\ 163, Avenue de Luminy, case 902, 13288 Marseille, France
}

\begin{abstract}
Recent results obtained by the H1 experiment at HERA are presented: searches for new physics using the full luminosity accumulated at high energy, measurements of the proton structure at low and high $Q^{2}$ and studies of hadronic final states.
\end{abstract}

\section{Introduction}

HERA is a unique electron- or positron-proton collider with a centre-of-mass energy of up to $320 \mathrm{GeV}$. The data taking extended over 15 years since 1992 and yielded an integrated luminosity of close to $0.5 \mathrm{fb}^{-1}$ for physics analyses. In March 2007 HERA completed data taking at high energy and started collisions at lower centre-of-mass energies of $225 \mathrm{GeV}$ and $275 \mathrm{GeV}$ in order to allow a direct measurement of the longitudinal structure function of the proton. The main physics avenues of the HERA program are illustrated in this paper with recent results obtained by the $\mathrm{H} 1$ collaboration in the following areas: searches for new physics proton structure measurements and studies of hadronic final states [1].

\section{Searches at the Energy Frontier}

\subsection{Measurement of events with isolated leptons at HERA}

The $\mathrm{H} 1$ data sample accumulated at high energy and corresponding to $0.5 \mathrm{fb}^{-1}$ enables the search for rare phenomena, with cross sections around or below $1 \mathrm{pb}$. One such process is the production of $W$ bosons, for which the total production cross section is around $1.3 \mathrm{pb}^{-1}$, calculated including NLO-QCD corrections [2]. If the $W$ boson decays leptonically, the corresponding events contain an energetic, isolated lepton and significant missing energy due to the escaping neutrino.

Such events have been observed at HERA by the H1 collaboration [3]. Moreover, an excess of events with large hadronic transverse momentum $P_{T}^{X}$ was reported after the first data taking period HERA I (1994-2000, $\left.118 \mathrm{pb}^{-1}\right)$, where 11 events are observed with $P_{T}^{X}>25 \mathrm{GeV}$ for a Standard Model (SM) expectation of $3.5 \pm 0.6$. The ZEUS collaboration also performed a search for this event topology, within an analysis aimed at a search for anomalous top production [4], but did not confirm the excess observed by H1. Here the results from the $\mathrm{H} 1$ analysis performed including all available data is presented [5]. A comparison with the most recent ZEUS analysis is also made [6].

Events with a lepton (electron or muon) transverse momentum above $10 \mathrm{GeV}$ in the angular range $5^{\circ}<\theta_{e}<140^{\circ}$ and with missing transverse momentum $P_{T}^{\text {miss }}>12 \mathrm{GeV}$ are selected. Extra background suppression criteria are also applied. A good agreement is observed with the SM predictions in the full phase space of the analysis. The H1 analysis measures 59 events for a SM expectation of $58 \pm 8.2$. The signal purity (dominated by the $W$ production) is $60-68 \%$ in the electron channel and $76-83 \%$ in the muon channel. This result therefore provides clear evidence of single $W$ boson production at HERA.

${ }^{*}$ Support from DESY is kindly acknowledged. 

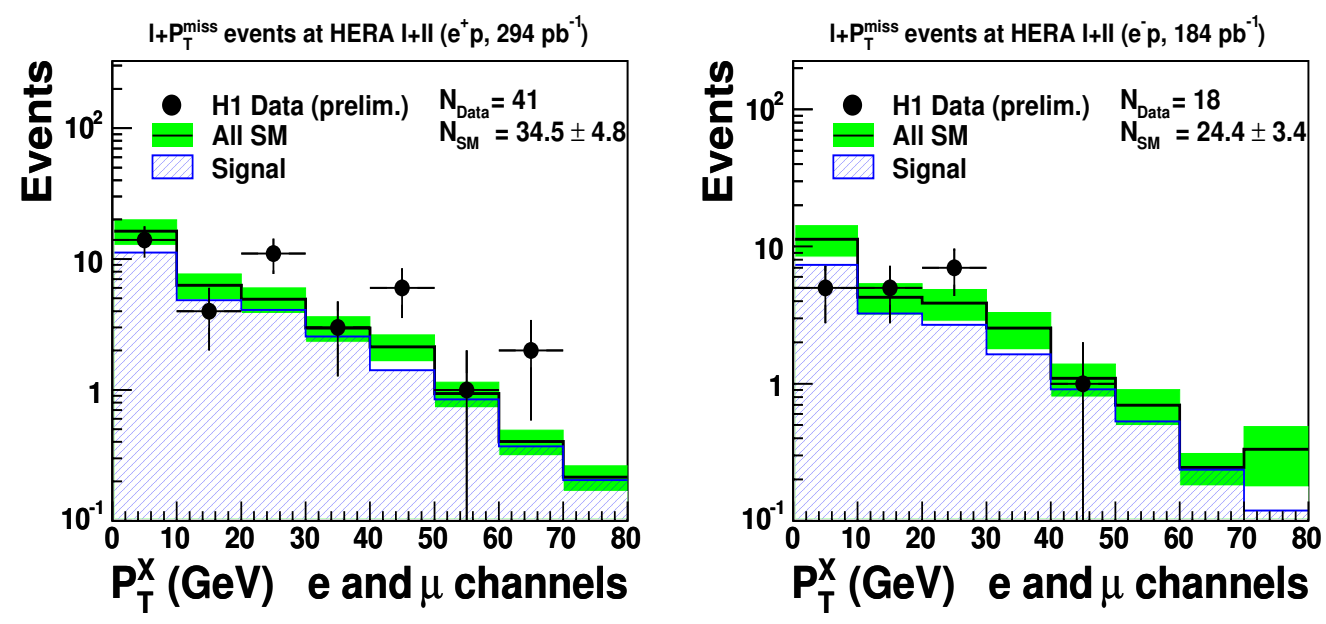

Figure 1: The distributions of the observed events as a function of $P_{T}^{X}$ in the H1 analysis in $e^{+} p$ data (left) and $e^{-} p$ data (right).

\begin{tabular}{lll|ccc}
\multicolumn{2}{c|}{$\begin{array}{c}\mathrm{e}^{ \pm} \mathrm{p} \text { Data Preliminary } \\
P_{T}^{X}>25 \text { GeV }\end{array}$} & $\begin{array}{c}\text { Electron } \\
\text { obs./exp. }\end{array}$ & $\begin{array}{c}\text { Muon } \\
\text { obs./exp. }\end{array}$ & $\begin{array}{c}\text { Combined } \\
\text { obs./exp. }\end{array}$ \\
\hline $\boldsymbol{e}^{+} \boldsymbol{p}$ & H1 & $294 \mathrm{pb}^{-1}$ & $11 / 4.7 \pm 0.9$ & $10 / 4.2 \pm 0.7$ & $21 / 8.9 \pm 1.5$ \\
& ZEUS & $228 \mathrm{pb}^{-1}$ & $1 / 3.2 \pm 0.4$ & $3 / 3.1 \pm 0.5$ & $4 / 6.3 \pm 0.9$ \\
\hline $\boldsymbol{e}^{-} \boldsymbol{p}$ & H1 & $184 \mathrm{pb}^{-1}$ & $3 / 3.8 \pm 0.6$ & $0 / 3.1 \pm 0.5$ & $3 / 6.9 \pm 1.1$ \\
& ZEUS & $204 \mathrm{pb}^{-1}$ & $5 / 3.8 \pm 0.6$ & $2 / 2.2 \pm 0.3$ & $5 / 6.0 \pm 0.9$
\end{tabular}

Table 1: The observed and expected numbers of events in the region $P_{T}^{X}>25 \mathrm{GeV}$ in $\mathrm{H} 1$ and ZEUS analyses.

The distribution of events in the H1 analysis as a function of $P_{T}^{X}$ is shown separately in $e^{+} p$ and $e^{-} p$ data samples in figure 1. This result indicates that the excess of events at large $P_{T}^{X}$ originates from the $e^{+} p$ data sample. The observations of H1 and ZEUS analyses at $P_{T}^{X}>25 \mathrm{GeV}$ are shown in table 1 . The excess observed by $\mathrm{H} 1$ in $e^{+} p$ data has a significance of about $3.0 \sigma$ but is not confirmed by the ZEUS analysis. In the $e^{-} p$ data sample, a good agreement with the SM is observed by both H1 and ZEUS. The different observations of H1 and ZEUS at large $P_{T}^{X}$ have been investigated and differences in the acceptance understood [7]. An analysis in a common phase space of H1 and ZEUS would allow the direct comparison and the combination of the data in order to exploit these events for further SM measurements of W production and investigations of new physics models.

\subsection{Multi-lepton events at HERA}

Within the Standard Model (SM) the production of multilepton events in ep collisions is possible mainly through photon-photon interactions, where quasi-real photons radiated from the incident electron and proton interact for producing a pair of leptons $\gamma \gamma \rightarrow \ell^{+} \ell^{-}$. The 

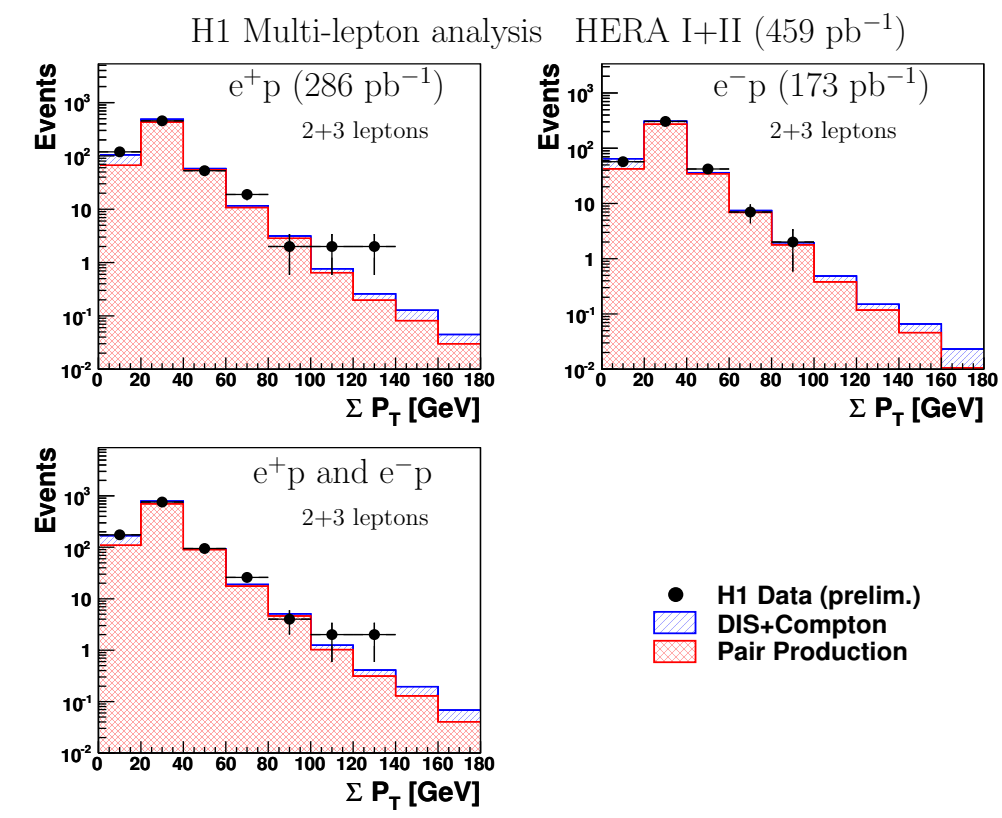

Figure 2: Distributions of the sum of the transverse momenta of the leptons in the H1 multi-lepton analysis.

H1 collaboration uses the full data sample at high energy to seach for events with several leptons (electrons or muons) [8]. Events with at least two leptons in the central region of the detector $20^{\circ}<\theta_{\ell}^{1,2}<140^{\circ}$ and with high transverse momenta $P_{T}^{1(2)}>10(5) \mathrm{GeV}$ are selected. All other leptons in the full detector acceptance are identified and the events are classified according to the lepton content. Data events are observed in the configuration ee, $e e e, e \mu, \mu \mu$ and $e \mu \mu$ in agreement with the predictions from the SM. In order to compare the di-lepton and the tri-lepton events, the scalar sum of the transverse momenta of the leptons in the event $\sum P_{T}$ is used. The distributions of $\sum P_{T}$ obtained after combining all channels are shown in figure 2 for $e^{+} p, e^{-} p$ and full data samples. Four events are observed with a scalar sum of lepton transverse momenta greater than $100 \mathrm{GeV}$, compared to a SM expectation of $1.9 \pm 0.4$. The four events with $\sum P_{T}>100 \mathrm{GeV}$ are observed in $e^{+} p$ collisions only, where the SM expectation is of $1.2 \pm 0.2$.

\subsection{Searches for leptoquarks at HERA}

HERA is an ideal machine for producing new bosons coupling to lepton and quarks. In a minimal model incorporating the SM internal and chiral symetries [9], 14 species of leptoquarks are predicted. In addition to charge, isospin and chirality, these new bosons are characterised by the fermion number $F$ which is an additive quantum number that reflects the particle $(+1)$ and antiparticle (-1) coupling content. The LQs produced in $e^{-} p$ collisions have predominantly $F=2$, whereas $F=0$ leptoquarks are mainly produced in $e^{+} p$ collisions. The significant increase in luminosity at HERA II, especially in $e^{-} p$ collisions, opened a new discovery window. The leptoquarks decays are seached for in the electron-jet 

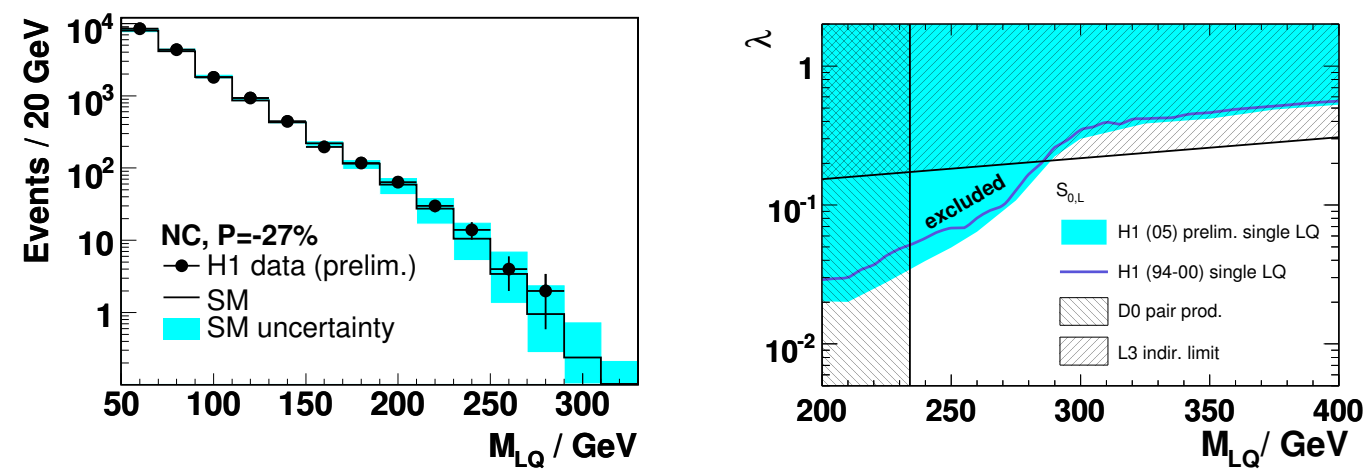

Figure 3: Exclusion domains in the plane of the leptoquark mass and its coupling to fermions $\lambda$. Limits from the present $\mathrm{H} 1$ analysis, based on $e^{-} p$ data $\left(92 \mathrm{pb}^{-1}\right)$, are compared with limits from LEP and Tevatron.

and neutrino-jet final states, corresponding to irreducible backgrounds from neutral and charged current DIS scattering [10]. Due to the chiral nature of LQ's, the $e$-beam polarisation enhances the sensitivity to certain species. An example of invariant mass spectrum obtained in electron-jet channel for negative $e$-beam polarisation is shown in figure 3 (left). No LQ signal is detected. Limits on the model parameter are deduced. An example of exclusion domain is shown in figure 3 (right), comparing also with limits obtained at LEP and Tevatron. The new results extend significantly the domains explored at HERA I. The increase in data in both $e^{+} p$ and $e^{-} p$ is expected to give rise to new opportunities to search for leptoquarks at HERA.

\subsection{Searches for matter substructure}

The fermion mass hierarchy is one of the greatest puzzles of the Standard Model (SM). It can naturally be explained if the SM fermions are composite, in which case excited states may exist and be produced at colliders. A minimal extension [11] of the SM that incorporates excited fermions introduces new parameters: the compositness scale $\Lambda$ (which reflects the range of the new confinement force) and the couplings $f$ and $f^{\prime}$ (corresponding to the weak $\mathrm{SU}(2)$ and electromagnetic $\mathrm{U}(1)$ sectors respectively).

Excited electrons and neutrinos can be produced in electron-proton collisions at HERA via the $t$-channel neutral current $e^{ \pm} p \rightarrow e^{*} X$ or charged current $e^{ \pm} p \rightarrow \nu^{*} X$ reactions.

For the excited neutrinos, the cross section is much larger in $e^{-} p$ collisions than in $e^{+} p$ collisions due to the helicity enhancement, specific to CC-like processes. The present search for $\nu^{*}$ uses a data sample corresponding to an integrated luminosity of $184 \mathrm{pb}^{-1}$ data sample, a factor of 12 larger than the previously published analyses at HERA [12]. The full data sample at highest energy $\left(\sqrt{s}=319 \mathrm{GeV}, 435 \mathrm{pb}^{-1}\right)$ is used for excited electron searches [13].

Excited neutrinos are searched for in the channels $\nu^{*} \rightarrow \nu \gamma, \nu Z, e W$ where the $\mathrm{W}$ and $\mathrm{Z}$ bosons are reconstructed in the leptonic and hadronic channels. The analysis covers $95 \%$ (85\%) of the total branching ratio for $f=-f^{\prime}\left(f=+f^{\prime}\right)$. The excited electrons are searched 

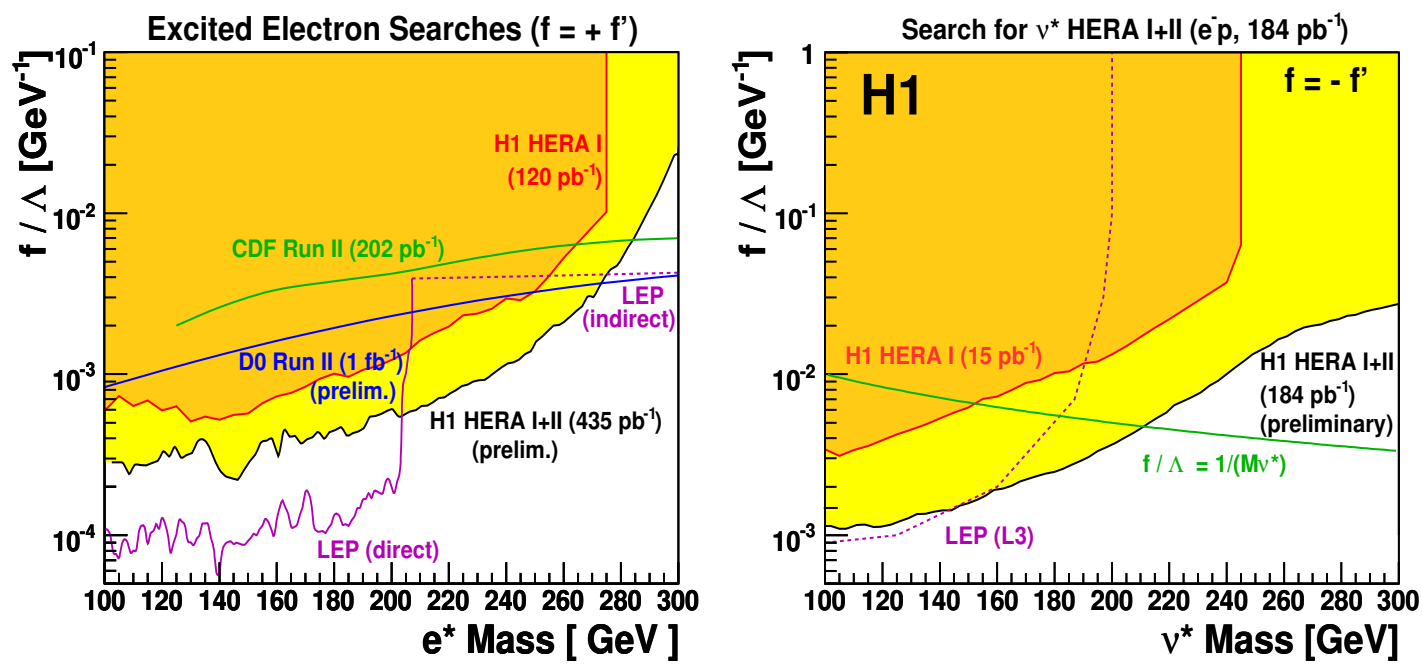

Figure 4: Exclusion domains for $f / \Lambda$ as a function of the excited state mass obtained for excited electrons (left, $f=+f^{\prime}$ ) and excited neutrinos (right, $f=-f^{\prime}$ )

in the channels $e^{*} \rightarrow e \gamma, e Z, \nu W$ where the $\mathrm{W}$ and $\mathrm{Z}$ bosons are reconstructed in the hadronic decay channel, for a $85 \%$ coverage of the $e^{*}$ decay width, independent of its mass. The signal is searched for as a peak in the invariant mass distributions. No significant deviation from the SM prediction is found and limits on the production of excited leptons are deduced. These limits on the cross section are translated into exclusion domains in the plane $(f / \Lambda$, $\left.M_{\nu *, e^{*}}\right)$. The result obtained for $e^{*}$ assuming $f=+f^{\prime}$ is shown in figure 4(left). For this configuration and assuming $f / \Lambda=1 / M_{e *}$, excited electrons with masses below $273 \mathrm{GeV}$ are excluded at $95 \% \mathrm{CL}$. The exclusion domain obtained for excited neutrinos for $f=-f^{\prime}$ (maximal $\gamma \nu \nu *$ coupling) is shown in figure 4(right). Assuming $f / \Lambda=1 / M_{\nu *}$, excited neutrinos with masses below $188 \mathrm{GeV}$ are excluded at 95\% CL. The present results greatly extend previous searched domains at HERA and confirm the unique HERA sensitivity for excited electrons and neutrinos with masses beyond the reach of LEP.

\section{Proton Structure Measurements with the Highest Precision}

\subsection{Measurements at high $Q^{2}$ and electroweak fits}

The increase in luminosity and the use of polarised electron or positron beams at HERA II creates new opportunities for proton structure measurements at high $Q^{2}$. The electroweak effects in the inclusive NC cross sections are measured for instance by comparing the $e^{+} p$ and $e^{-} p$ data. The difference is proportional to the parity violating structure function $x F_{3}$. A measurement of this structure function performed by combining H1 and ZEUS data is shown in figure 5 .

The combined QCD-electroweak fit [14] has been performed using HERA II data [15], taking advantage of the electron or positron beam longitudinal polarisation. In this fit, the strategy is to leave free in the fit the EW parameters together with the parameterisation 

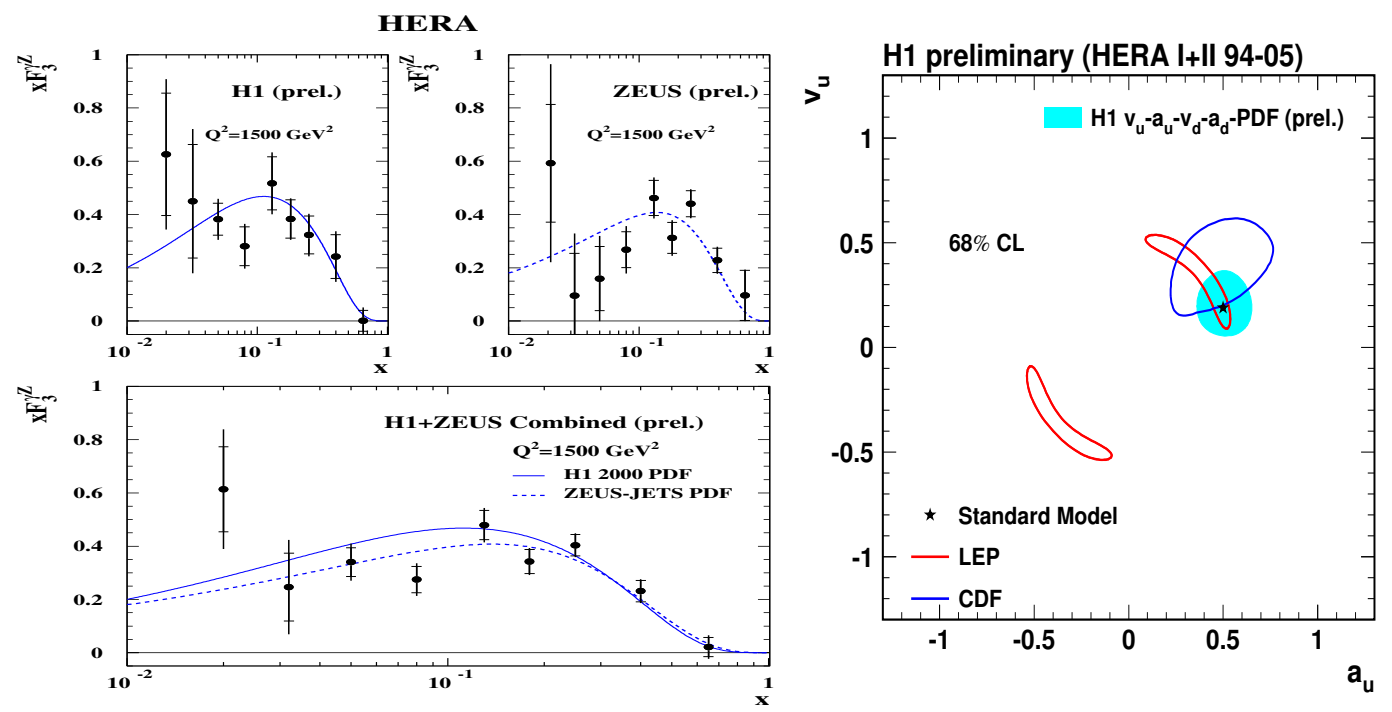

Figure 5: Left: Measurement of the parity violating structure function $x F 3$. Right: Axial and vector couplings of the $u$-quark measured from the combined electroweak-QCD fit at HERA and compared with measurements from LEP and Tevatron.

of the parton distribution functions. Due to the $t$-channel electron-quark scattering via $Z$ bosons, the DIS cross sections at high $Q^{2}$ are sensitive to light quark axial $\left(a_{q}\right)$ and vector $\left(v_{q}\right)$ coupling to the $Z$. This dependence includes linear terms with significant weight in the cross section which allow the determination of not only the value but also the sign of the couplings. In contrast, the measurements at the $Z$ resonance (LEP1 and SLD) only access $a v$ or $a^{2}+v^{2}$ combinations. Therefore there is an ambiguity between axial and vector couplings and only the relative sign can be determined. In addition, since the flavour separation for light quarks cannot be achieved experimentally, flavour universality assumptions have to be made. The Tevatron measurement [16] of the Drell-Yan process allows to access the couplings at an energy beyond the $Z$ mass resonance, where linear contributions are significant. The measurements of the $u$-quark couplings obtained at HERA, LEP and the Tevatron are shown in figure 5(right). The new $\mathrm{H} 1$ measurement has an improved precision compared to previous published values, corresponding for instance to a factor two for $v_{u}$. The data to be collected at the Tevatron and HERA as well as the use of polarised $e^{ \pm}$beams at HERA open interesting oportunities for the light quarks couplings measurements in the near future.

\subsection{High precision studies at low $Q^{2}$}

The measurements of deeply inelastic scattering at HERA reach very low values of photon virtuality and extend to domains where perturbative QCD cannot be applied. The transition region from photoproduction $\left(Q^{2} \simeq 0\right)$ to DIS is studied with high precisions using the low angle detectors and special runs with either high trigger rates to improve statistical and systematical errors or shifted collision vertex in order to access lower scattering angles and therefore smaller $Q^{2}$ values. 
The H1 collaboration has recentlly released the final results of the measurement of DIS in the range $0.2<Q^{2}<12 \mathrm{GeV}^{2}$ and at very low $x$ from $4 \cdot 10^{-6}$ to 0.02 [17]. A precision of $2 \%$ to $4 \%$ is achieved and various models of the transition from real to virtual photonproton interactions are tested. The lowest $x$ region is sensitive to the longitudinal structure function $F_{L}$ since this region corresponds to the highest $y$ domain, where the contribution of $F_{L}$ term to the reduced cross section is favoured, according to $\sigma_{r}=F_{2}-\left(y^{2} / Y^{+}\right) F_{L}$ where $Y^{+}=1+(1-y)^{2}$. The longitudial structure function is a fundamental form factor of the proton, related to the scattering of longitudinally polarised photons of quarks, which can only take place if gluons are radiated during the interaction. As a consequence, $F_{L}$ give access to the gluon content of the proton $F_{L}\left(x, Q^{2}\right) \sim \alpha_{s} x g\left(x, Q^{2}\right)$. The measurement of the high $y$ region is therefore important for the precision measurement of the proton content.

\subsection{Measurements at high $y$ and direct determination of $F_{L}$}
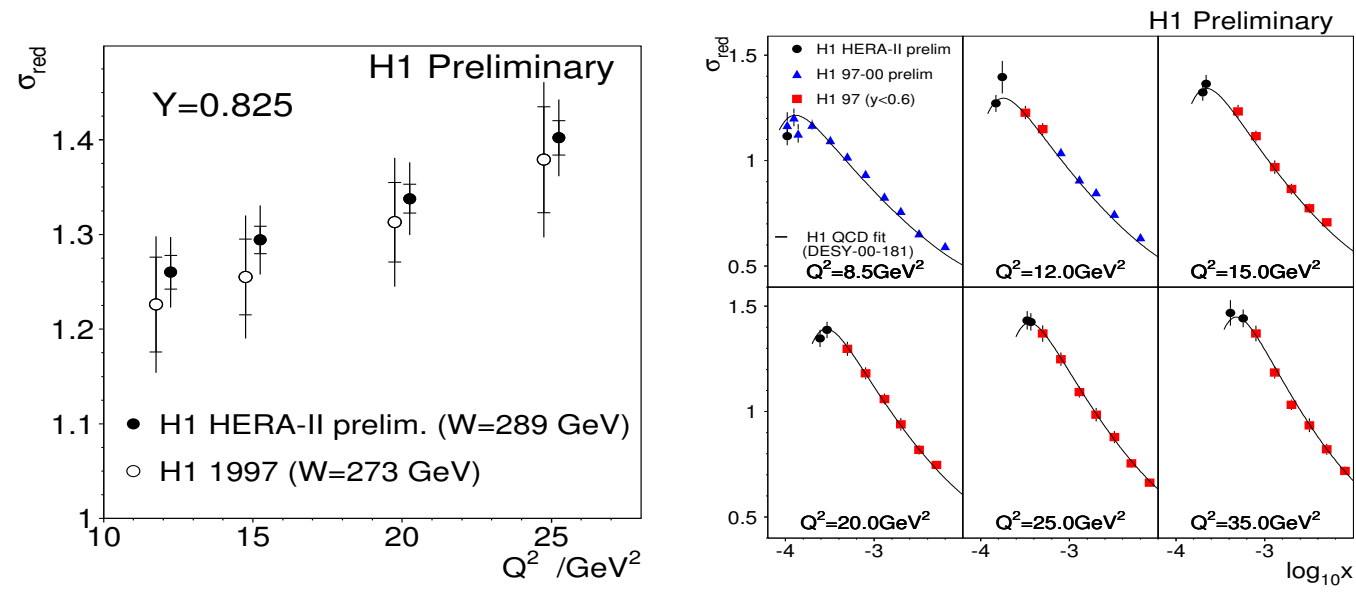

Figure 6: Reduced cross section measurements of DIS process at high $y$ in the range $8<$ $Q^{2}<35 \mathrm{GeV}^{2}$, for $y=0.825$ (left) and as a function of $x$ and $Q^{2}$ (right).

In the proton rest frame, the inelasticity can be expressed as a function of the energies of the incoming and scattered electrons: $y=\left(E_{e}^{0}-E_{e}\right) / E_{e}^{0}$. The high $y$ regime therefore corresponds to events where the final state electron has a low energy. The measurement of this kinematic region implies a special experimental approach since the trigger and the background rejection are difficult. In particular, the background from photoproduction induced by the misidentification of hadrons as electrons has to be subtracted. In a new analysis [18], H1 collaboration uses the electron candidate charge measurement, in order to subtract this background using data. The measurement of the reduced cross section as a function of $Q^{2}$ for $y=0.825$ is shown in figure 6(left) and displays a significant improvement in precision compared with the previous publication. The new cross section measurement as a function of $x$ and $Q^{2}$ is shown in figure 6(right). It dramatically improves the precision in the regions at low $x$ where the $F_{L}$ contribution is expected to be significant.

The $F_{L}$ structure function can be directly measured if the reduced cross section $\sigma \sim$ $F_{2}\left(x, Q^{2}\right)+f(y) F_{L}\left(x, Q^{2}\right)$ is measured for different $y$ values at fixed $x$ and $Q^{2}$. This can 


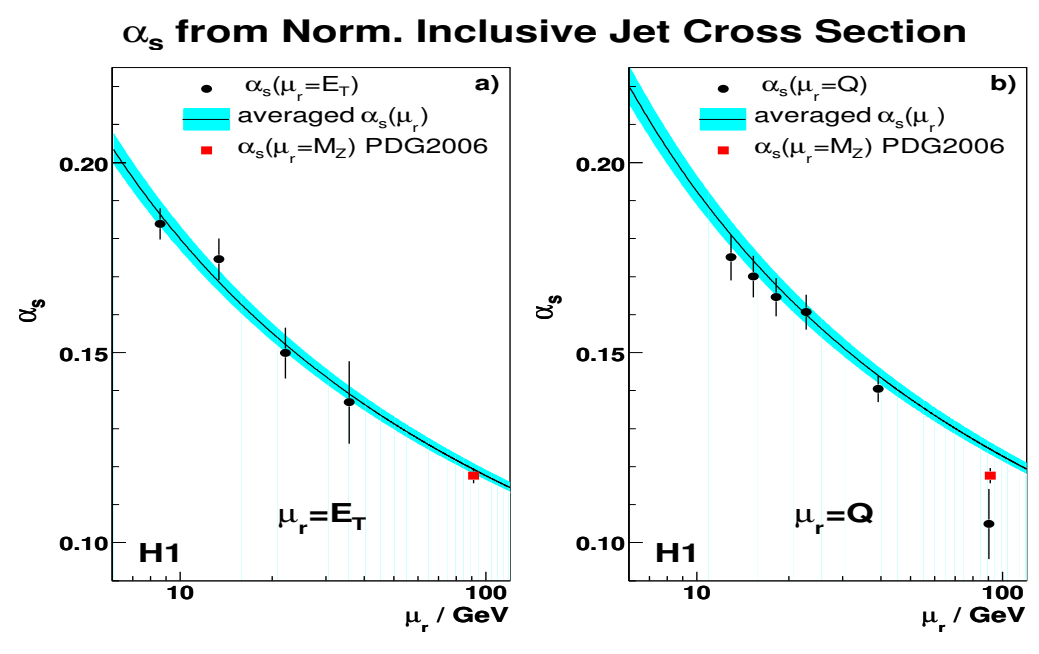

Figure 7: Results for the fitted values of a) $\alpha_{s}\left(\mu_{r}=E_{T}\right)$ averaged over all $Q^{2}$ regions, and b) $\alpha_{s}\left(\mu_{r}=Q\right)$ averaged over all $E_{T}$ regions. The error bars denote the total experimental uncertainty for each data point. The solid curve shows the result of evolving $\alpha_{s}\left(M_{Z}\right)$ averaged from all $Q^{2}$ and $E_{T}$ regions, with the band denoting the total experimental uncertainty. The world average from PDG is also shown.

be achieved if the beam energies are varied. The last three months of HERA running were dedicated to a run a lower centre-of-mass energy. This data will be used for the first direct measurement of $F_{L}$ at low $x$ and will provide a new constraint on the gluon density in the proton [19].

\section{QCD Studies in a Clean High Energy Laboratory}

\subsection{Jet Production at high $Q^{2}$ and determination of $\alpha_{\mathrm{S}}$}

The measurement of jet production allows to test QCD since it can be related to gluon radiation of to gluon-photon collisions. A new measurement of jet production using HERA I data has been recently published [20].

The inclusive jet cross section is measured in DIS with a $Q^{2}>100 \mathrm{GeV}^{2}$. For $\mathrm{NC}$ DIS events in the range $0.2<y<0.7$ and in a given $Q^{2}$ bin, the normalised inclusive jet cross section is defined as the average number of jets within $-1.0<\eta^{\mathrm{Lab}}<2.5$ per event. The double differential cross section is measured in six bins of $Q^{2}$ and four bins of $E_{T}$. The normalised jet cross section is used to extract a precise measurement of the strong coupling. To study the scale dependence of $\alpha_{s}$, the six data points with different $Q^{2}$ at a given $E_{T}$ are used together, and four values of $\alpha_{s}\left(E_{T}\right)$ are extracted. The results are shown in figure $7 \mathrm{a}$, where the running of the strong coupling is also clearly observed. Finally, all 24 measurements are used in a common fit of the strong coupling, which yields

$$
\alpha_{s}\left(M_{Z}\right)=0.1193 \pm 0.0014 \text { (exp.) }{ }_{-0.0030}^{+0.0047} \text { (th.) } \pm 0.0016 \text { (pdf) }
$$

with a fit quality of $\chi^{2} / \mathrm{ndf}=28.7 / 23$. The dominating source of error is the renormalisation scale dependence which is used to estimate the effect of missing higher orders beyond NLO 

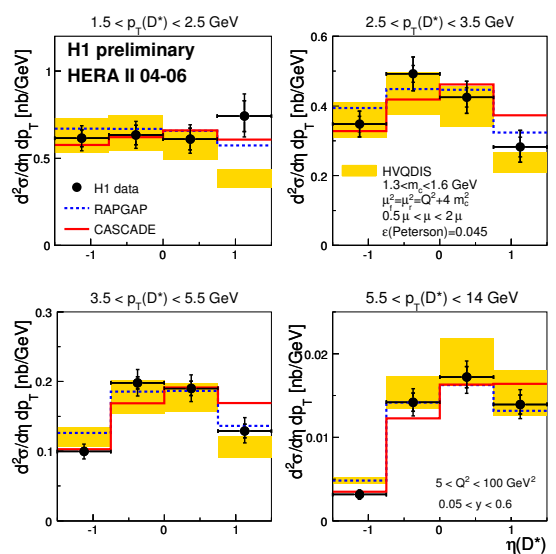

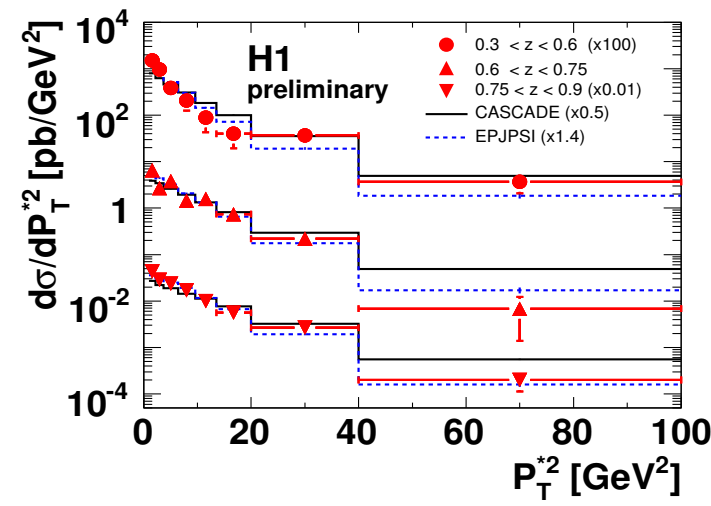

Figure 8: Left: the measured double differential cross section for $D^{*}$ production as a function of pseudorapidity and transverse momentum. Right: the measurement of the double differential cross section for $J / \Psi$ production as a function of the transverse momentum and the fraction of virtual photon momentum carried by the $J / \Psi$ meson.

in the pQCD prediction. This result shows a level of experimental precision competitive with $\alpha_{s}$ determinations from other recent jet production measurements at HERA [21] and is in good agreement with the world average 0.1176 \pm 0.0020 (PDG 2006) [22].

\subsection{Charm production}

The measurement of the production of charm particles is particularly important since the theoretical predictions are expected to be robust due to the large scale in the calculation provided by the charm quark mass. Using the HERA II data, the H1 collaboration performed two new measurements which significantly improve the precision in this area.

First, the $D^{*}$ production cross section is measured using a data sample collected by H1 experiment in the years 2004 to 2006 and corresponding to an intergated luminosity of $226 \mathrm{pb}^{-1}$. The analysis covers the kinematic region $5<Q^{2}<100 \mathrm{GeV}^{2}$ and $0.05<y<0.6$. The visible range for the $\mathrm{D}^{*}$ meson is restricted to $P_{T}\left(D^{*}\right)>1.5 \mathrm{GeV}$ and $\left|\eta\left(D^{*}\right)\right|<1.5$ where more than $10,000 \mathrm{D}^{*}$ mesons are reconstructed. The preliminary measurement of the double differential cross section is shown in figure 8(left). Precise measurements of single and double differential inclusive cross sections of $\mathrm{D}^{*}$ meson production are compared to LO predictions and a NLO calculation in the 'massive scheme'.

Second, the electroproduction of $J / \Psi$ mesons is measured with high precision using HERA II data accumulated in the period 2004-2006 and corresponding to $258 \mathrm{pb}^{-1}$. Charmonium production in DIS is modelled in non-relativistic QCD as a convolution between the short distance effects driven by the $Q^{2}$ and long distance matrix elements associated to the $J / \Psi$ emergence. Single and double differential cross sections and angular distributions are measured and compared to predictions from Monte Carlo programs implementing the colour singlet model at leading order. The predictions have to be scaled by large factors and, even after this scaling, they do not describe all aspects of the data, as illustrated in figure 8 (right). The increased experimental precision therefore challenges the theoretical 

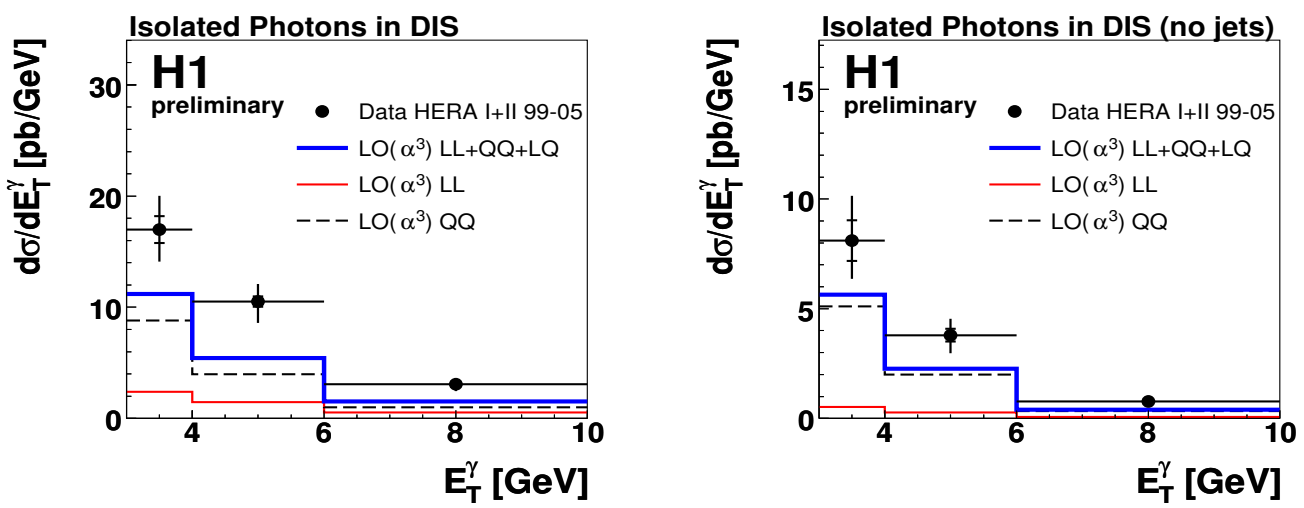

Figure 9: The cross section for isolated photons production in DIS as a function of photon transverse momentum for the inclusive analysis (left) and for events with no extra hadronic jet (right).

predictions in this area.

\subsection{Isolated photon production}

The production of isolated photons in hadronic collisions can bring important information about the hard scattering since the photons act as a clean probe, unaffected by the hadronisation uncertainties specific to hadrons emerging from the interaction. The production of isolated photons is studied in DIS with a four-momentum transfer squared of $4<Q^{2}<150 \mathrm{GeV}^{2}$ and a squared mass of the hadronic system $W_{X}^{2}>2500 \mathrm{GeV}^{2}$ using a total integrated luminosity of $226 \mathrm{ps}^{-1}$ including HERA II data [23]. Photons are identified in the transverse momentum range $3<E_{T}^{\gamma}<10 \mathrm{GeV}$ and in the pseudorapidity range $-1.2<\eta_{\gamma}<1.8$ using a multivariate analysis based on the cluster shower shapes in the calorimeter. Measured cross sections are compared with predictions from a LO calculation and MC models including the contribution of radiation from quarks (QQ) and from electrons (LL). The result is shown as a function of the photon transverse momentum in figure 9(left). The QQ contribution is enhanced when no jet is allowed in the events, as seen in figure 9 (right). The LO predictions underestimate the measurements by up to a factor of two, in particular at low $Q^{2}$. The understanding of this difference may greatly profit from the NLO calculation, which exists at present only for events with an additional jet [24].

\subsection{Deeply virtual Compton scattering}

Information beyond the longitudinal momentum distributions, including transverse momentum distribtutions and correlations between partons inside the proton can be obtained via processes where the proton remains intact after the interaction though the transverse momentum of the transfer is non-zero. Such a process is the diffractive scattering of a virtual photon $\gamma^{*} p \rightarrow \gamma p$, also called elastic Deeply Virtual Compton Scattering (DVCS). This process is accessed at HERA via the reaction $e p \rightarrow e p \gamma$, which also receives an important contribution from the Bethe-Heitler $(\mathrm{BH})$ process, where the photon is radiated off the electron in the final state. The reaction is measured by the $\mathrm{H} 1$ collaboration using $e^{+} p$ 

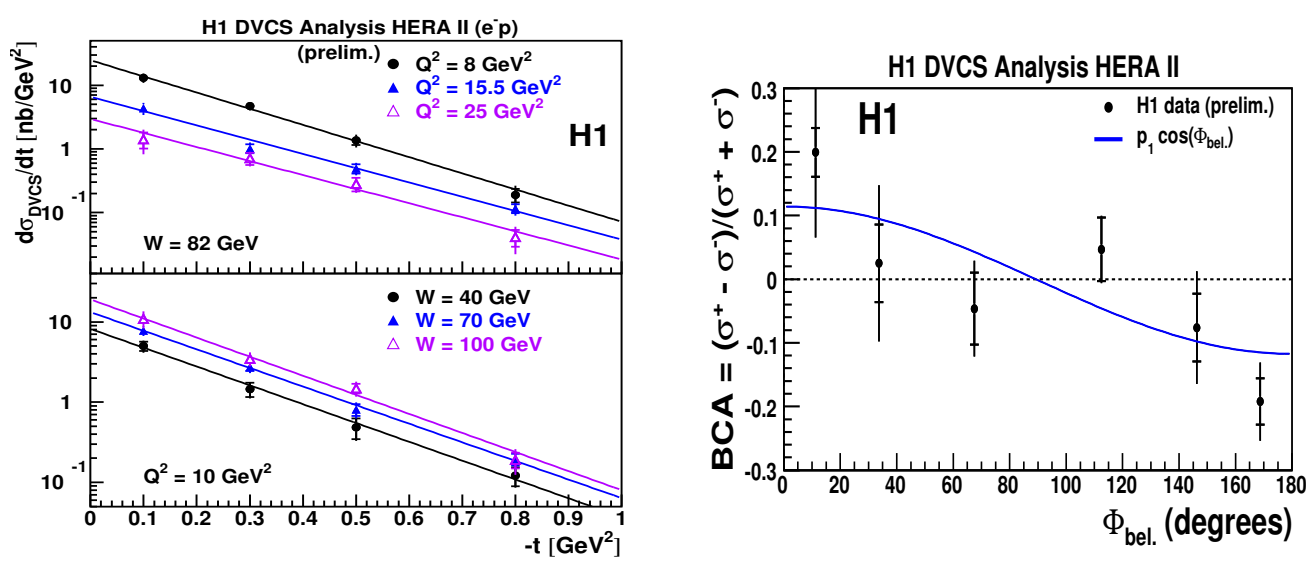

Figure 10: Left: the measurement of the DVCS cross section as a function of $t \mathrm{n}$ various $Q^{2}$ and $W$ bins. Right: the measured beam charge asymmetry as a function of the angle $\Phi$ between the production plane and the scattering plane.

$\left(146 \mathrm{pb}^{-1}\right)$ and $e^{-} p$ data $\left(146 \mathrm{pb}^{-1}\right)$ [25]. Cross sections are measured as a function of the virtuality of the exchanged photon $Q^{2}$ and the centre-of-mass energy of the $\gamma^{*} p$ system, W, in the kinematic domain $6.5<Q^{2}<80 \mathrm{GeV}^{2}, \quad 30<W<140 \mathrm{GeV}$ and $|t|<1 \mathrm{GeV}$, where $t$ denotes the squared momentum transfer at the proton vertex. The cross sections are measured differentially in $t$ for different $Q^{2}$ and $W$ values, as shown in figure 10(left). Using an exponential $t$-dependence parameterisation $d \sigma / d t \sim \exp -b t$, a global exponential $t$-slope parameter is derived to be $5.45 \pm 0.19 \pm 0.34$. The $b$ parameter is related to the mean impact parameter and can be related to a transverse size of the proton of $0.65 \pm 0.02 \mathrm{fm}$ at $x=1.2 \cdot 10^{-2}$. This is lower than the estimated proton radius $R_{p}=0.862(12) \mathrm{fm}$, as expected, since DVCS probes the core of the proton with no account of the peripheral soft structure. The cross sections are compared with NLO calculation based on generalised parton distributions and are found to be in good agreement. The dipole model is also investigated. The data is found to be in good agreement with the geometric scaling predicted by this model. Finally, the beam charge asymmetry (BCA) of the DVCS cross section is measured as a function of the angle $\Phi$ between the production plane and the scattering plane. The first measurement of this observable in colision mode is shown in figure 10(right). The BCA is proportional with the interference between the DVCS and BH cross sections and therefore directly accesses the generalised parton distribution functions. A dependence in $\cos \Phi$ is established $B C A=p_{1} \cos \Phi$ with $p_{1}=0.17 \pm 0.03($ stat $) \pm 0.05$ (sys) after the experimental deconvolution.

\section{Outlook}

The end of data taking opened a new, exciting era of final analyses at HERA ${ }^{\mathrm{a}}$. The searches for new physics are now performed on the full available statistics corresponding to a luminosity of $0.5 \mathrm{fb}^{-1}$. The excess of events with isolated electrons or muons, missing transverse

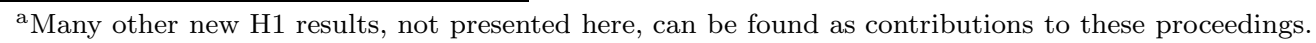


momentum and a prominent hadronic jet measured by $\mathrm{H} 1$ persists over more than ten years and constitutes one of the remaining puzzles of the HERA program. The precise measurement of the proton structure continues and benefits from the significant increase in statistics. The measurements of DIS at low $Q^{2}$ are complemented by a new, dedicated analysis of the high $y$ domain which will constrain the gluon density at low $x$. The measurements of hadronic final states allow an improved precision in $\alpha_{s}$ determination. Using the HERA II data, heavy quark measurements enter a precision domain and challenge further the theoretical calculations. The "final touch" of HERA is the run at low energy, which will allow the measurement of the longitudinal structure function and a new constraint on the gluon density at low $x$.

High precision measurements will be achieved at HERA in the following years using the full data sample and by combining $\mathrm{H} 1$ and ZEUS data sets. The physics contained in this data will be revealed in the next years and will constitute the legacy of HERA for the LHC and beyond.

\section{References}

[1] Slides: http://indico. cern. ch/contributionDisplay py?contribId=2\&sessionId=2\&conf Id=9499

[2] K.-P. O. Diener, C. Schwanenberger, and M. Spira, Eur. Phys. J. C 25 (2002) 405, and references therein.

[3] V. Andreev et al. [H1 Collaboration], Phys. Lett. B561 (2003) 241, [hep-ex/0301030].

[4] S. Chekanov et al. [ZEUS Collaboration], Phys. Lett. B 559 (2003) 153, [hep-ex/0302010].

[5] H1 Collaboration, Conference Note available at http://www-h1.desy.de/h1/www/publications/htmlsplit/H1prelim-07-063.long.html

[6] Katherine Korcsak-Gorzo, these proceedings.

[7] Ytsen de Boer, these proceedings.

[8] Gerhard Brandt, these proceedings.

[9] W. Buchmüller, R. Rückl and D. Wyler, Phys. Lett. B 191 (1987) 442; Erratum-ibid. B 448 (1999) 320.

[10] Anna Dubak, these proceedings.

[11] K. Hagiwara, D. Zeppenfeld and S. Komamiya, Z. Phys. C 29 (1985) 115; U. Baur, M. Spira and P. M. Zerwas, Phys. Rev. D 42 (1990) 815; F. Boudjema, A. Djouadi and J. L. Kneur, Z. Phys. C 57 (1993) 425.

[12] C. Adloff et al. [H1 Collaboration], Phys. Lett. B 525 (2002) 9.

[13] Nguyet Trihn, these proceedings.

[14] A. Aktas et al. [H1 Collaboration], hep-ex/0507080.

[15] Li Gang, these proceedings

[16] D. Acosta et al. [CDF Collaboration], Phys. Rev. D 71, 052002 (2005) [hep-ex/0411059].

[17] Andrea Vargas, these proceedings

[18] Natasa Raicevic, these proceedings

[19] Max Klein, these proceedings

[20] A. Aktas et al. [H1 Collaboration], arXiv:0706.3722 [hep-ex], to be published in Phys. Lett. B.

[21] S. Chekanov et al. [ZEUS Collaboration], Submitted to Phys. Lett. B [hep-ex/0701039].

[22] W. M. Yao et al. [Particle Data Group], J. Phys. G 33 (2006) 1.

[23] Katharina Müller, these proceedings.

[24] A. Gehrmann-De Ridder et al., Nucl. Phys. B 578 (2000) 326 [hep-ph/0003082].

[25] Laurent Schoffel, these proceedings. 